\title{
Maturation-dependent microstructure length scale in the corpus callosum of fixed rat brains by magnetic resonance diffusion-diffraction
}

\author{
Jun-Cheng Weng ${ }^{\text {a }}$, Jyh-Horng Chen ${ }^{\mathrm{a}}$, Li-Wei Kuo ${ }^{\mathrm{a}}$, Van J. Wedeen ${ }^{\mathrm{b}}$, Wen-Yih I. Tseng ${ }^{\mathrm{c}, \mathrm{d}, *}$ \\ ${ }^{a}$ Department of Electrical Engineering, National Taiwan University, Interdisciplinary MRI/MRS Laboratory, Taipei, Taiwan, Republic of China \\ ${ }^{\mathrm{b}}$ MGH Martinos Center for Biomedical Imaging, Harvard Medical School, Charlestown, MA, USA \\ ${ }^{\mathrm{c} C e n t e r}$ for Optoelectronic Biomedicine, College of Medicine, National Taiwan University, Taipei, Taiwan 100, Republic of China \\ ${ }^{\mathrm{d}}$ Department of Medical Imaging, National Taiwan University Hospital, Taipei, Taiwan, Republic of China \\ Received 19 June 2006; accepted 31 August 2006
}

\begin{abstract}
Techniques capable of assessing microstructure length scale are potentially useful in probing the integrity of biologic tissue at the microscopic level. Although the magnetic resonance (MR) diffusion-diffraction technique has been proposed for years, its realization in an undissected brain has not been reported on. In this study, validation of this method in a phantom simulating a series of repeated sheets of water with regular spacing was first performed. The same technique was applied to the corpus callosum of fixed rat brains of different ages (range, 21-84 days). The phantom was constructed with a pile of transparencies immersed in water doped with Gd-DTPA. The measured signal showed diffraction-like coherence peaks, the modulation of which was influenced by the gap distance and the center-to-center distance of the adjacent gaps. The measured distances were consistent with the actual values. In five 84-day-old rats, the diffusion length scale derived from the diffractogram was highly reproducible. In the course of brain maturation, the measured size decreased with age. Electron microscopy showed that axons on day 21 were smaller in diameter and less myelinated as compared with those on day 84 . Progressive decrease in the diffusion length scale observed during brain maturation might reflect a gradual decrease in transmembrane permeability due to myelination. In conclusion, MR diffusion-diffraction can be observed in the corpus callosum of fixed rat brains. This technique might be useful in probing the status of myelination in the development of disease.
\end{abstract}

(C) 2007 Elsevier Inc. All rights reserved.

Keywords: MRI; Diffusion; Diffraction; Microstructure; Diffractograms

\section{Introduction}

Techniques capable of assessing the length scales of microstructures are potentially useful in studying the integrity of biologic tissue at the microscopic level. Two magnetic resonance (MR) techniques, $k$-space diffraction and $q$-space diffraction, have been proposed to probe the power spectra of the density function of microstructures. The $k$-space diffraction technique, first proposed by Mansfield and Grannell [1] and later demonstrated by Barrall et al. [2], utilizes raw data of MR images in the spatial frequency domain, called $k$-space, to obtain the power spectra of the density function. It requires spatial resolution comparable with microstructure

* Corresponding author. Center for Optoelectronic Biomedicine, College of Medicine, National Taiwan University, No 1, Jen-Ai Road, Section 1, Taipei, Taiwan 100, Republic of China. Tel.: +886 22312 3456x8758; fax: +886 223926922 .

E-mail address: wytseng@ha.mc.ntu.edu.tw (W.-Y.I. Tseng). size and adequate cell-to-background contrast. Such images are difficult to obtain from biologic tissue. The $q$-space approach, proposed by Callaghan et al. [3], probes the microstructure based on the translational diffusion of water molecules. Typically, a series of MR signals is measured with different diffusion-sensitive gradients. Each gradient generates modulation of transverse magnetization with a specific spatial frequency that corresponds to a unique $q$ value. The sampled signals at different $q$ values form a diffractogram from which the length scale of the microstructure can be derived. Since the source of signal modulation in the diffractogram comes from the restrictive diffusion in the organized microenvironment and since the average diffusion length during typical measurement duration is close to a cell size, this technique is more suitable for biologic studies.

Although the diffusion-diffraction technique has the potential to characterize microstructure sizes, its realization in biologic tissue is challenging. The difficulty arises from the 
fact that cells are not perfectly aligned [4] as well as from the fact that water molecules exchange between intracellular and extracellular compartments due to transmembrane permeability $[5,6]$. These factors can smear out or distort the diffraction pattern. Although there are reports showing coherence features in erythrocyte suspensions [7-11], to the best of our knowledge, no report has been made to reveal coherence diffraction patterns from undissected brains.

In this study, we first tested the diffusion-diffraction technique in a phantom constructed to approximate a series of repeated sheets of water with regular spacing. We demonstrated the effects of diffusion time and diffusion gradient direction on the diffraction pattern. We then applied the same technique to the corpus callosum of fixed rat brains with optimum parameters. The corpus callosum consists of commissural axonal fibers connecting bilateral cerebral cortices. Axons in the middle portion of the corpus callosum pack tightly and run parallel to each other. Myelin sheath wrapping the axon forms a natural barrier to hinder the water exchange between intracellular and extracellular water. These features make the corpus callosum an ideal model for diffusiondiffraction experimentation. To test the sensitivity of this technique, we studied the corpus callosum of rats of different ages to demonstrate the change in the diffusion length scale during the course of brain maturation. The results were compared with $T_{1}$ and $T_{2}$ values and electron microscopy.

\section{Materials and methods}

The experiments were performed in a 3-T MR imaging (MRI) Biospec system (Bruker Companies, Ettlingen, Germany). We used a high-performance gradient coil mounted on the table of the 3-T magnet (inner diameter $=6$ $\mathrm{cm}$; maximal gradient strength $=1000 \mathrm{mT} / \mathrm{m}$ ) and a quadrature coil with an inner diameter of $3.5 \mathrm{~cm}$ for radiofrequency transmission and reception.

\subsection{Phantom experiment}

We constructed a phantom consisting of a stack of transparencies to approximate sheets of water with regular spacing. One hundred thirty transparencies were piled. The size of each transparency was $15 \times 25 \mathrm{~mm}$, and the thickness of the whole stack was $13 \mathrm{~mm}$. The margins of each transparency were not sealed so that the water molecules within each gap can diffuse to nearby gaps through the interconnecting space surrounding the margins. To achieve better image contrast, we immersed the phantom in water doped with Gd-DTPA (Magnevist, Schering, Allemagne, Germany) at a concentration of about $0.5 \mathrm{mM}$. To eliminate air bubbles in the solution, we placed the phantom in a hot bath at $70^{\circ} \mathrm{C}$ for $12 \mathrm{~h}$ before scanning.

\section{2. q-Space diffraction experiment}

We used a stimulated echo diffusion-weighted sequence to obtain $q$-space diffractograms. Images having the following parameters were acquired: repetition time (TR)/ echo time $(\mathrm{TE})=1016 / 20 \mathrm{~ms}$; field of view $(\mathrm{FOV})=25 \mathrm{~mm}$; slice thickness $=8 \mathrm{~mm}$; matrix size $=32$; and number of excitations $(\mathrm{NEX})=5$. The diffusion gradient duration $(\delta)$ was $6 \mathrm{~ms}$, and the diffusion time ( 4 ) was $1000 \mathrm{~ms}$. A series of diffusion gradients $(g)$ was applied, with the strengths ranging from 0 to $950 \mathrm{mT} / \mathrm{m}$, achieving maximal diffusion sensitivity: $b_{\max }=\gamma^{2} g^{2} \delta^{2}(\Delta-\delta / 3)=2.3 \times 10^{6} \mathrm{~s} / \mathrm{mm}^{2}$. The $q$ values increased incrementally with the gradient strength $g$ according to the relation $q=\gamma g \delta(2 \pi)^{-1}$. To obtain sufficient samples in different regimes of $q$, we performed nonuniform sampling. Five increments (i.e., 3, 5, 10, 30 and $50 \mathrm{mT} / \mathrm{m}$ ) were used in five ranges of gradient strengths $(0-100,100$ $150,150-200,200-350$ and $350-950 \mathrm{mT} / \mathrm{m}$, respectively). Sixty-six diffusion-weighted images (DWIs) corresponding to $66 q$ values were obtained in about $6 \mathrm{~h}$.

To study the effect of $\Delta$ on the diffractogram, we repeated the experiment with five $\Delta$ values at 20, 50, 200, 600 and $1000 \mathrm{~ms}$; to study that of diffusion gradient directions on the diffractogram, we acquired three sets of data, with the diffusion gradients applied separately in the read $(X)$, phase $(Y)$ and through-plane $(Z)$ directions.

\section{3. q-Space diffraction pattern analysis}

A region of interest (ROI) slightly smaller in size than the phantom surface was selected from DWIs, and signal intensities of the pixels within the ROI were averaged. The averaged signal intensities in each spectral series were normalized by the values of the central peak in the same series.

As described by Callaghan et al. [12], the diffractogram obtained from a system comprising repeated cells with finite wall thickness is a harmonic function modulated by a sincsquare envelope. From the curve of the sinc-square envelope, the reciprocal of the $q$-distance from the origin to the first node or between two neighboring nodes

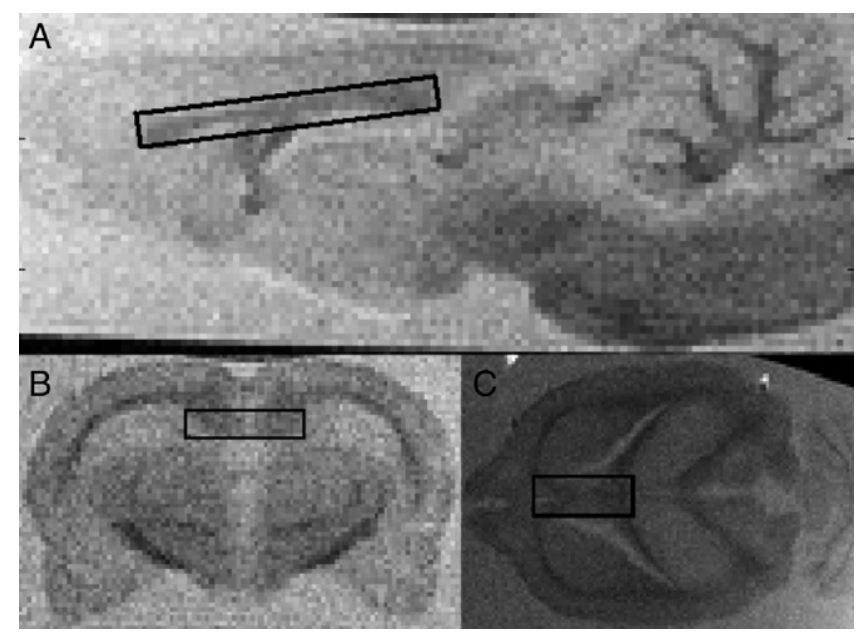

Fig. 1. $T_{1}$-weighted images of rat brains in the sagittal (A) and coronal (B) planes acquired with relatively low resolution to localize a high-resolution axial image (C) parallel to the middle portion of the corpus callosum enclosed by a rectangle. The $q$-space diffraction pattern is observed in the region of the corpus callosum with various diffusion gradients applied perpendicular to the axial plane. 


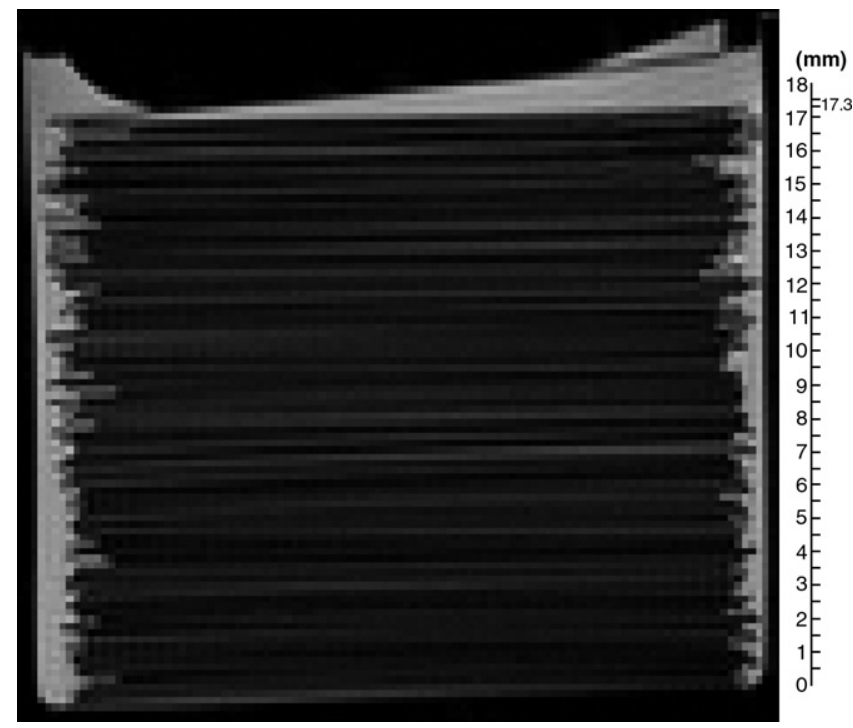

Fig. 2. $T_{1}$-weighted image of a phantom system consisting of a pile of transparencies. The phantom is immersed in water doped with Gd-DTPA. As indicated in the figure, the total thickness of the phantom immersed in the solution is $17.3 \mathrm{~mm}$.

corresponds to the gap distance. From the harmonic function curve, the reciprocal of the $q$-distance from the origin to the first peak or between two neighboring peaks corresponds to the distance between adjacent gap centers.

\subsection{Rat experiment}

All the procedures of the animal experiment adhered to the Guidelines for Care and Use of Experimental Animals by the Laboratory Animal Center of the National Taiwan University. We studied four male Wistar rats aged 21, 28, 56 and 84 days; their body weights were $48,73,330$ and $400 \mathrm{~g}$. $T_{1}, T_{2}$ and diffusion-diffraction were measured from the corpus callosum of each rat. To test procedural reproducibility, we performed MR diffusion-diffraction on five 84day-old adult rats.

Wistar rats were euthanized with an intraperitoneal injection of sodium pentobarbital and were perfused using formaldehyde-fixed liquid after they failed to respond to a hard tail pinch. The rat brains were taken out on a plastic box and immersed in the formaldehyde-fixed liquid. The box was then placed into the quadrature coil for scanning. $T_{1}$-weighted images in the sagittal and coronal planes were obtained to localize the imaging plane parallel to the middle portion of the corpus callosum (Fig. 1).

\section{5. q-Space diffraction experiment in rats}

After the imaging plane was defined, we used a spinecho diffusion-weighted sequence to obtain a $q$-space diffractogram. The parameters were as follows: TR/ $\mathrm{TE}=1500 / 67 \mathrm{~ms} ; \mathrm{FOV}=22 \mathrm{~mm}$; slice thickness $=1 \mathrm{~mm}$; and matrix size $=16$. The diffusion gradients perpendicular to the imaging plane were applied with $\delta=4 \mathrm{~ms}$ and $\Delta=60$ ms. Magnitudes of diffusion gradients were incremented from 0 to $850 \mathrm{mT} / \mathrm{m}$, reaching maximal diffusion sensitivity $b_{\max }=4.85 \times 10^{4} \mathrm{~s} / \mathrm{mm}^{2}$. In the rats aged between 21 and 84 days, five increments $(3,5,10,30$ and $50 \mathrm{mT} / \mathrm{m})$ were used for five ranges of gradient strengths $(0-100,100-150,150$ $200,200-350$ and $350-850 \mathrm{mT} / \mathrm{m}$, respectively). With six

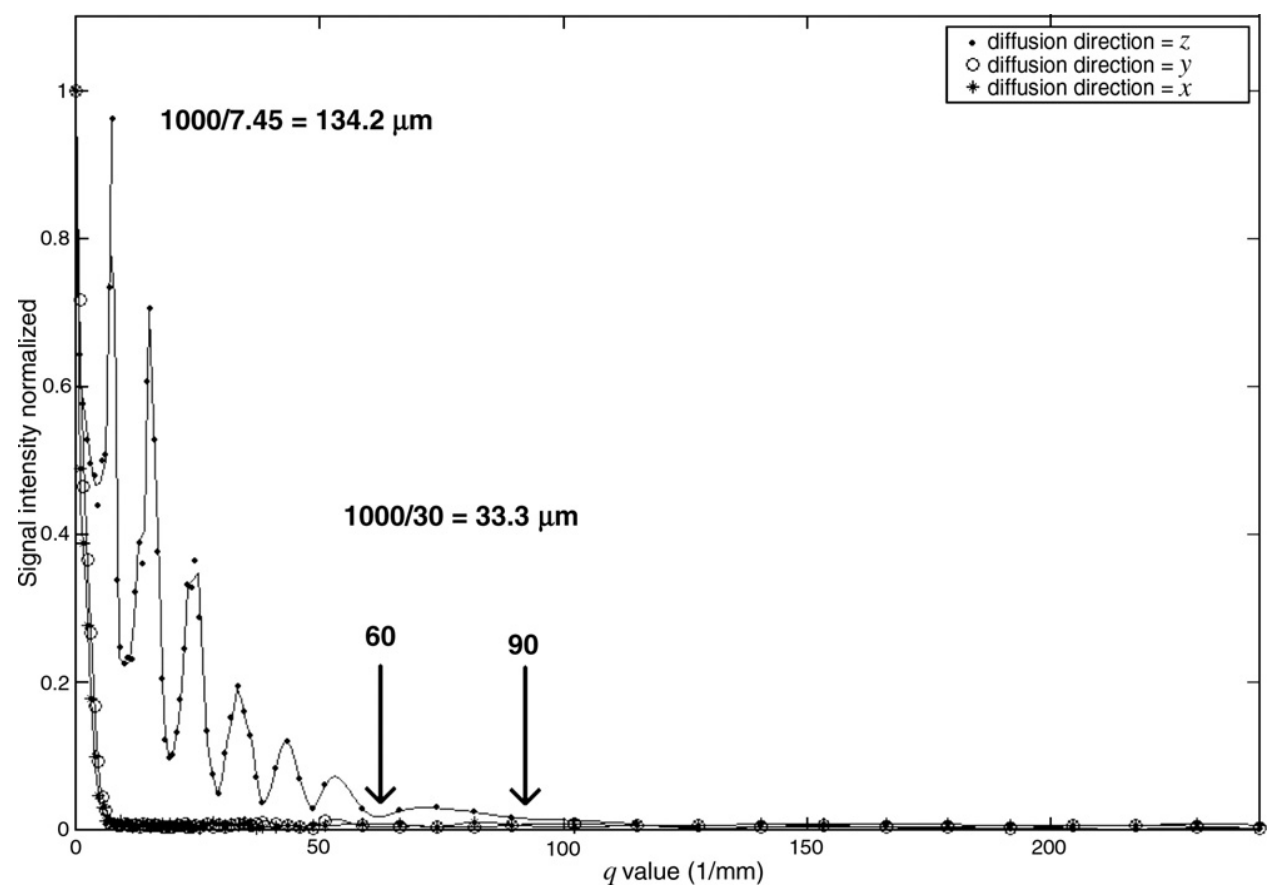

Fig. 3. Nuclear MR $q$-space diffraction technique measured in three orthogonal diffusion gradient directions. Only the measurement in the through-plane direction (i.e., perpendicular to the planes of the transparencies) shows the diffraction pattern. The measured value of the gap distance and that of the center-tocenter distance are 33.3 and $134.2 \mu \mathrm{m}$, respectively. The gap distance is calculated from the $q$-distance between two neighboring nodes of the sinc-square envelope curve at 60 and $90 \mathrm{~mm}$. 


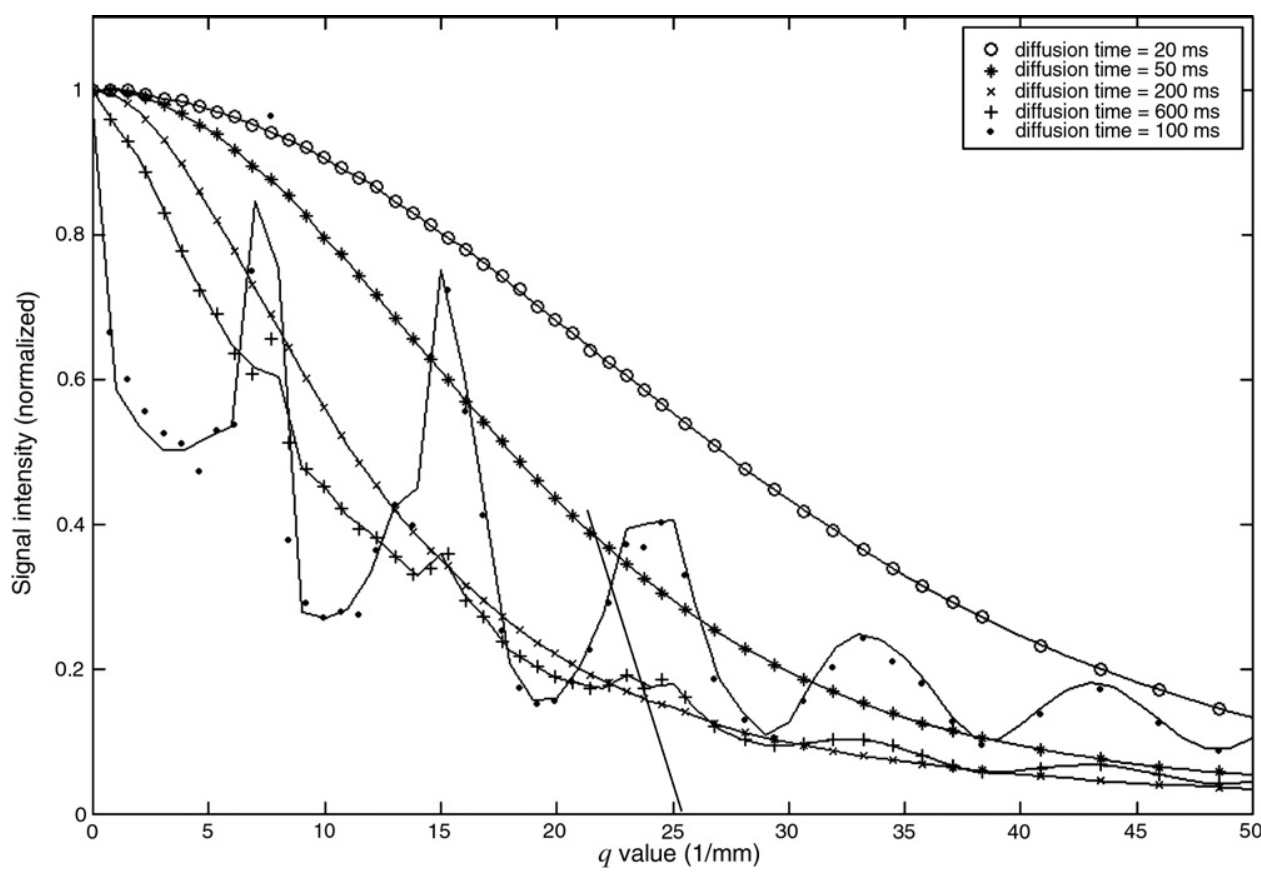

Fig. 4. The effect of $\Delta$ on the diffusion diffractogram in a phantom. The diffraction pattern was observed when the $\Delta$ values were 600 and $1000 \mathrm{~ms}$ but disappeared when the $\Delta$ values were 20,50 and $200 \mathrm{~ms}$ because the average displacement of water molecules $(2 D \Delta)^{-1 / 2}$ was $<a$, where $D$ is the diffusion coefficient and $a$ is the gap distance.

NEX for each data acquisition, 64 DWIs corresponding to $64 q$ values were obtained in about $2.5 \mathrm{~h}$.

An ROI covering the middle portion of the corpus callosum was first defined on high-resolution $T_{1}$-weighted images that had the same FOV and slice position as did
DWIs, and pixel intensities on DWIs within the ROI were selected and averaged. The measured signal intensities in each spectral series were normalized by the value of the central peak in the same series. $T_{1}$ and $T_{2}$ values in the same ROI of the corpus callosum were also measured using true-

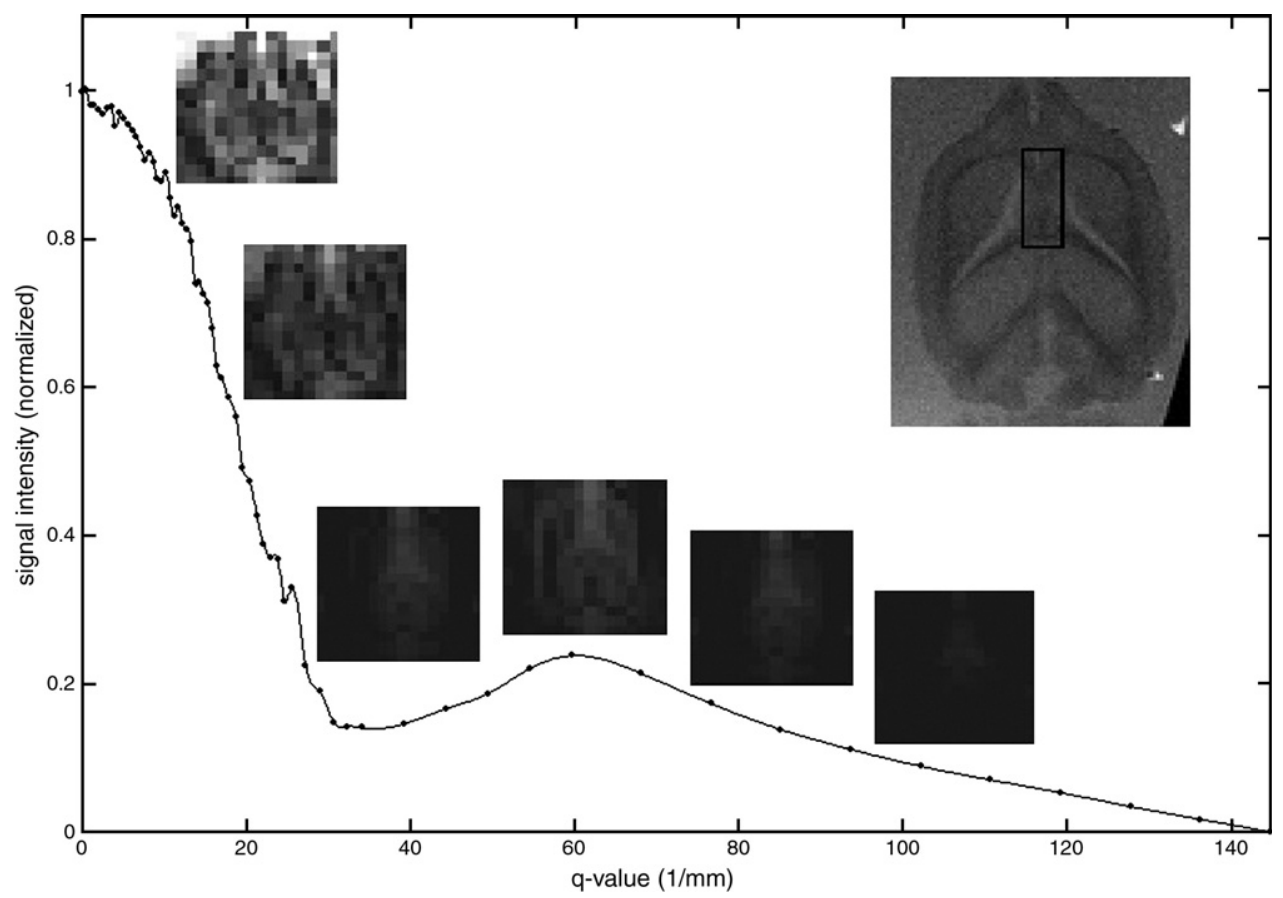

Fig. 5. A diffraction curve measured from a series of DWIs. The six images shown were acquired at $q$ values of $0,20,30,60,80 \mathrm{and}_{100} \mathrm{~mm}^{-1}$. Note that signal intensity in the corpus callosum decreases from 0 to $30 \mathrm{~mm}^{-1}$, rises at $60 \mathrm{~mm}^{-1}$ and decreases again at 80 and $100 \mathrm{~mm}^{-1}$. 


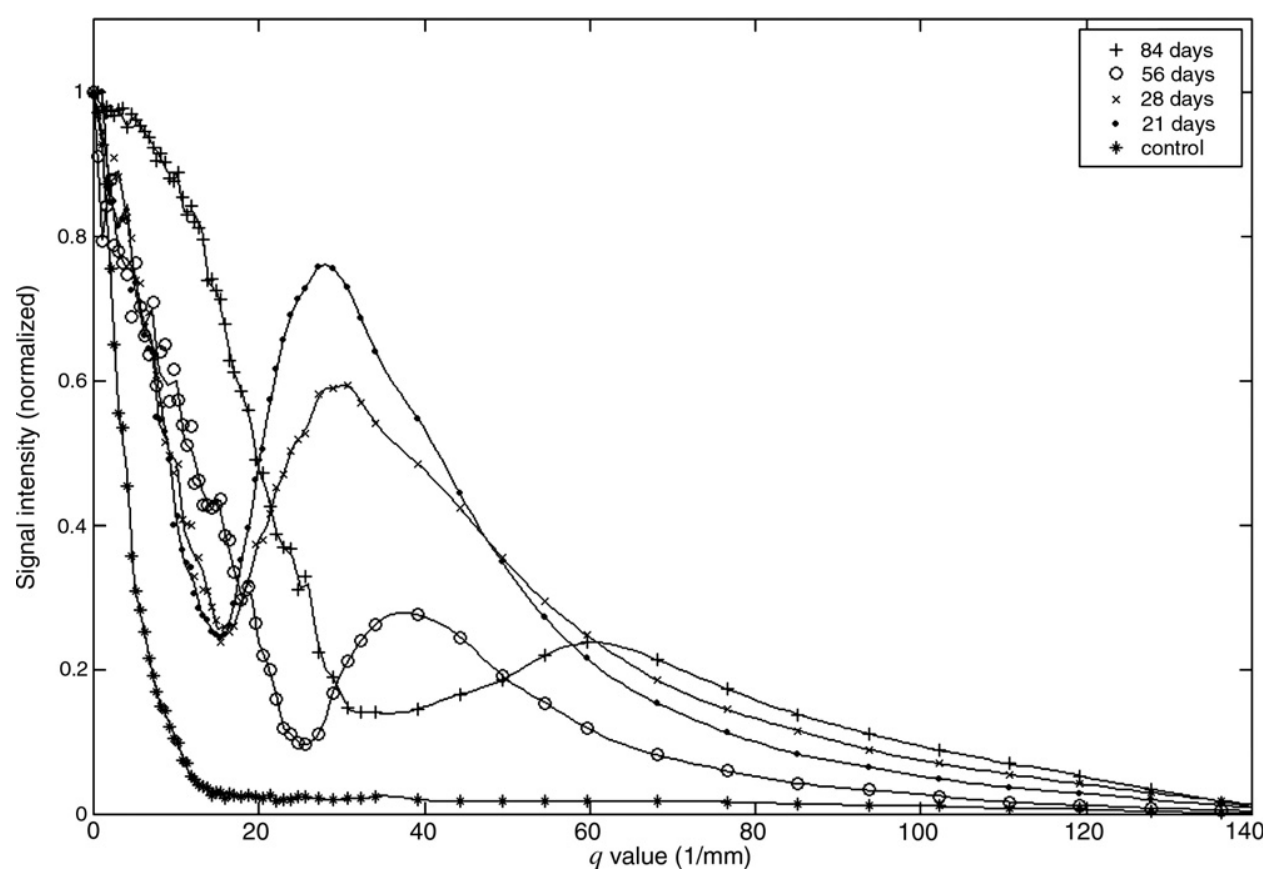

Fig. 6. Diffusion diffractogram observed in the corpus callosum of rats that were between 21 and 84 days old. As the brain matured, the position of the first node shifted progressively to the higher $q$ values, indicating a gradual decrease in the displacement range of water molecules.

FISP (fast imaging with steady-state free precession) and multislice multiecho sequences [13].

\section{Results}

The thickness of the 130 dry transparencies, measured with a high-precision vernier caliper (0- to 100-mm model, Accu-Check Instrument Service, OH, USA), was $13.0 \mathrm{~mm}$. The total thickness of the phantom immersed in the solution measured $17.3 \mathrm{~mm}$ on the $T_{1}$-weighted image (Fig. 2). From these two values, the average thickness of a transparency was $100 \mu \mathrm{m}$, the distance of a gap between two nearby transparencies was $34.1 \mu \mathrm{m}$ and the distance of the adjacent gap centers was $134.1 \mu \mathrm{m}$.

\section{1. q-Space diffractogram of the phantom}

Fig. 3 shows three curves of signal intensities versus $q$ in three orthogonal directions. The diffractogram was observed only when the diffusion gradient was applied in the direction perpendicular to the surface of transparencies. From the diffractogram, the second side lobe of the sinc-square-like envelope was identified. Two nodes beside the side lobe measured 60 and $90 \mathrm{~mm}$; the gap distance of transparencies was $33.3 \mu \mathrm{m}$ [i.e., $(90-60)^{-1} \mathrm{~mm}$ ]. From the $q$-distance
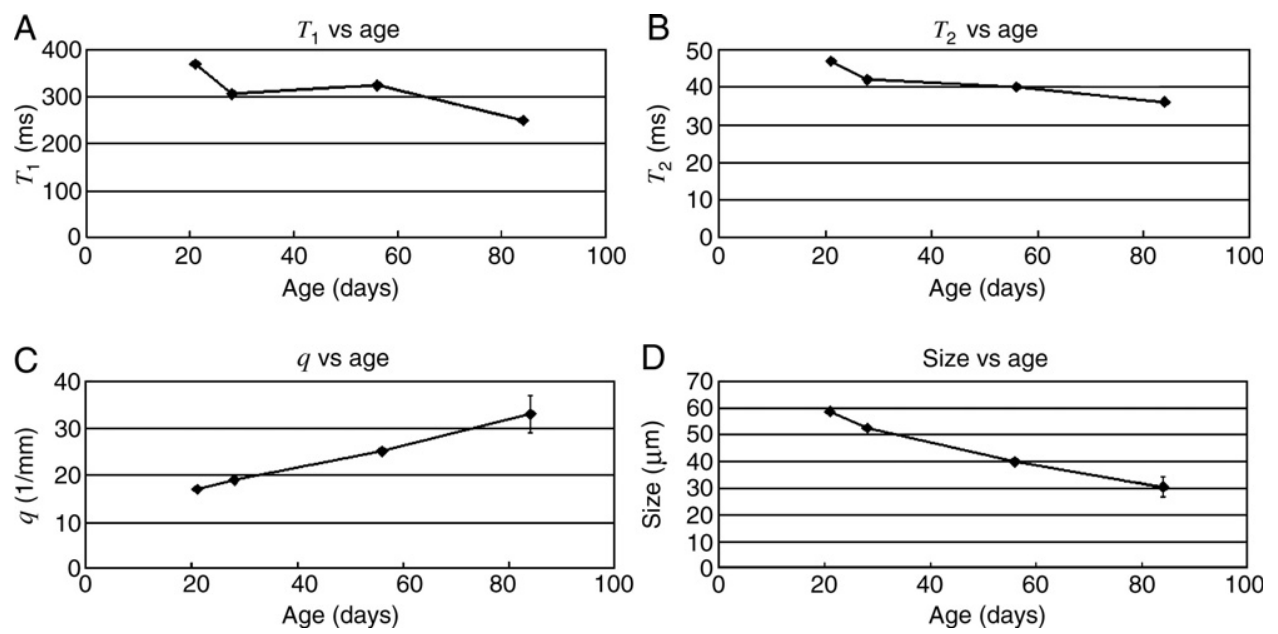

Fig. 7. Changes in $T_{1}$ (A), $T_{2}$ (B), $q$ value of the first node (C) and the derived microstructure size (D) of the corpus callosum during brain maturation. From day 21 to day 84, $T_{1}$ decreased from 369 to $249 \mathrm{~ms}$ and $T_{2}$ decreased from 47 to $36 \mathrm{~ms}$ (A and B). The measured diffusion length scale decreased more rapidly, from 58.8 to $30.7 \mu \mathrm{m}$ (D). The error bars in Panels (C) and (D) are the standard deviations of $q$ and size, respectively, in five adult rats. 
between the origin and the first peak at $7.45 \mathrm{~mm}$, the centerto-center distance of the adjacent gaps was $134.2 \mu \mathrm{m}$ [i.e., $(7.45)^{-1} \mathrm{~mm}$.

Fig. 4 shows five curves of signal intensities versus $q$ corresponding to five $\Delta$ values. Given the gap distance of our phantom $a=34.1 \mu \mathrm{m}$ and the diffusion coefficient of water molecules $D=2 \times 10^{-3} \mathrm{~mm}^{2} / \mathrm{s}$, diffractograms were expected to appear when $\Delta>a^{2} / 2 D=290 \mathrm{~ms}$. Indeed, these five curves show a transition from a Gaussian-like pattern when the $\Delta$ values were 20,50 and $200 \mathrm{~ms}$ to a diffraction pattern when the $\Delta$ values were 600 and $1000 \mathrm{~ms}$.

\subsection{Diffusion diffractogram of the rat corpus callosum}

To obtain the optimum parameters for a diffraction pattern in the rat corpus callosum, we tried different combinations of $\delta$ and $\Delta$. We chose $\delta=4 \mathrm{~ms}$ to obey the narrow gradient-pulse condition. In mature rats, diffraction patterns were clearly seen when $\Delta$ was in the range of 30 $100 \mathrm{~ms}$, the pattern being stretched to a higher $q$ regime as $\Delta$ decreased. When $\Delta>150 \mathrm{~ms}$, the diffraction patterns were too noisy to be observed due to the long TE.
In adult rats, the diffraction pattern of the corpus callosum was best observed when $\Delta=60 \mathrm{~ms}$ and $\delta=4 \mathrm{~ms}$ and the gradient direction was applied perpendicular to the axial view of the corpus callosum. A series of DWIs at different $q$ values and the resulting diffusion-diffraction curve are shown in Fig. 5. The diffraction pattern was compatible with the sinc-square envelope curves observed in the phantom study. Although the spectral intensities were not consistent between the studies due to different gain settings, the first nodes of the spectra can be identified reliably. From the curves, we determined the reciprocal of the $q$-distance between the origin and the first node to indicate the microstructure size of the corpus callosum.

\subsection{Reproducibility and maturation-dependent length scales of the corpus callosum}

A reproducibility study on five 84-day-old adult rats showed that the microstructure size was $30.67 \pm 3.79 \mu \mathrm{m}$. In the course of brain maturation, the diffraction pattern shifted to a high $q$ regime as age increased (Fig. 6). $T_{1}$ and $T_{2}$ values of the corpus callosum showed a moderate decrease from day

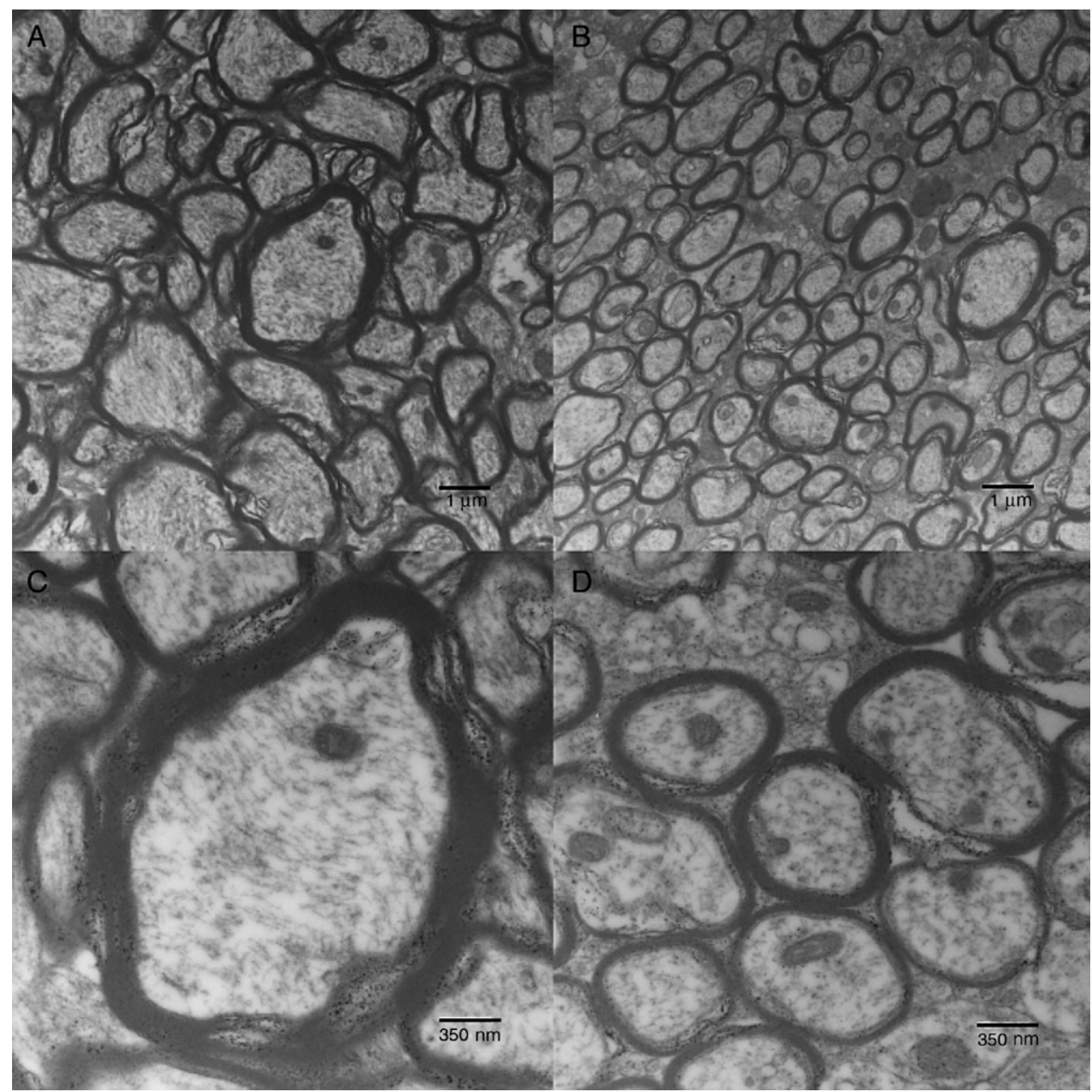

Fig. 8. Electron microscopic images of axonal fibers in the corpus callosum of an adult rat on day 84 (A and C) and a young rat on day 21 (B and D). The axonal fibers in the adult rat measure about $3 \mu \mathrm{m}$ and have well-developed myelination (A and $\mathrm{C}$ ). In comparison, the axonal fibers in the young rat are smaller, about $1 \mu \mathrm{m}$, and show minimal myelination (B and D). 
21 to day 84 (Fig. 7A and B). The measured length scale of the corpus callosum decreased more rapidly, from $58.8 \mu \mathrm{m}$ on day 21 to $30.7 \mu \mathrm{m}$ on day 84 (Fig. 7C and D).

To verify that the diffraction pattern was not caused by gradient interaction, we investigated the diffusion data acquired from the phantom and the rat brain. In the phantom experiment, three orthogonal diffusion gradients were applied separately. The diffraction pattern was observed only when the diffusion gradient was applied in the direction perpendicular to the surface of the transparencies. The signal showed monotonic signal decay when the diffusion gradients were applied in directions parallel to the surface of the transparencies (Fig. 3). In the rat brain study, the diffraction pattern was observed in the ROI enclosing the corpus callosum, whereas monotonic signal decay was observed in the water solution surrounding the rat brain (Fig. 6, the control curve).

\subsection{Electron microscopy}

Fig. 8 shows electron microscopic images of the corpus callosum in an adult rat on day 84 and a young rat on day 21. The axonal fibers in the adult rat were surrounded by thick myelin sheaths, and the averaged diameter of the axon measured about $3 \mu \mathrm{m}$ (Fig. 8A and C). The axonal fibers in the young rat showed minimal myelination, and the averaged diameter of the axon measured about $1 \mu \mathrm{m}$ (Fig. 8B and D).

\section{Discussion}

We demonstrated that MR diffusion-diffraction is observable in the corpus callosum of fixed rat brains. The technique was first tested in a phantom. When $\Delta$ was sufficiently large (i.e., $\Delta>a^{2} / 2 D$ ), the measured signal showed a diffraction pattern. The gap distance and centerto-center distance measured from the pattern were consistent with the values obtained from the high-precision vernier caliper and $T_{1}$-weighted images. Having verified the use of the technique in the phantom study, we applied the same technique to the corpus callosum of fixed rat brains. The diffusion length scale measured from the diffractogram was reproducible, and it decreased progressively in the course of brain maturation. Our results suggest that the measured length scale of the corpus callosum reflects the average range of molecular displacement that is possibly affected by the transmembrane permeability of microstructures.

\subsection{Diffusion diffractogram of the phantom}

Although the diffusion-diffraction obtained from the phantom yielded correct values of the gap distance and center-to-center distance, the diffractogram deviated from a typical diffraction pattern in three ways: (1) there was a gradual rise of the diffractogram toward a lower $q$ regime; (2) the coherence peaks in the second side lobe of sincsquare envelope were not shown; and (3) the shape of the envelope was similar to a Gaussian-like distribution rather than to a sinc-square function. It is instructive to discuss these deviations.

In a system containing $N$ repeated cells with spacing $b$, when $\Delta$ is infinite, the water molecules will have chances to arrive at the barriers and be reflected. The echo attenuation function $E_{\infty}(q)$ can be described as the power spectrum of a density function $|S(q)|^{2}$ times the convolution of the reciprocal lattice and the Fourier transform of a sharp truncation envelope, $\sin c^{2}(\pi q N b)$ [14], such that:

$E_{\infty}(q)=\left|S_{0}(q)\right|^{2}\left[\sin c^{2}(\pi q N b) \sum_{n=0}^{\infty} \delta(q-n / b)\right]$.

In the case of finite $\Delta, N b$ is much larger than the diffusive distance and the truncation is no longer sharp and takes the form of a gently decaying envelope. Therefore, the term $\sin$ $c^{2}(\pi q N b)$ in Eq. (1) is replaced by the Fourier spectrum of a diffusive envelope $\exp \left(-4 \pi^{2} q^{2} D_{\mathrm{p}} \Delta\right)$.

$E_{\Delta}(q) \approx\left|S_{0}(q)\right|^{2}\left[\exp \left(-4 \pi^{2} q^{2} D_{\mathrm{p}} \Delta\right) \sum_{m} \delta(q-m / b)\right]$,

where $D_{\mathrm{p}}$ is the coefficient of long-range diffusion reflecting the permeability of the cell walls. The Fourier spectrum of the diffusive envelope broadens the coherence peaks. Therefore, the diffractogram is an array of broadened coherence peaks modulated by the power spectrum of the density function of the gap $\left|S_{0}(q)\right|^{2}$ and the peak width is determined by the width of $\exp \left(-4 \pi^{2} q^{2} D_{\mathrm{p}} \Delta\right)$. As alluded to by Callaghan [14], the rise of our diffractogram toward a lower $q$ regime is the result of aliasing from the convolution of the broadened coherence peaks and the reciprocal lattice. This broadening effect also smears out the coherence peaks in the second side lobe of our diffractogram and makes them indistinguishable. Nonetheless, the second and third nodes beside the second side lobe can still be identified to obtain the gap distance.

Since the transparencies were immersed in water and were kept balanced by gravity force and hydrostatic pressure, the gaps between the transparencies were probably not uniform. This variability in gap distance would blur the sinc-square envelope to become a Gaussian-like distribution.

\subsection{Diffractogram of the rat corpus callosum}

Although we have shown that MR diffusion-diffraction can accurately measure the microstructure sizes in our phantom, as discussed above, there are substantial blurring effects on the coherence peaks and sinc-square envelopes. Implementation of this technique and interpretation of the results are much more challenging in a biologic system. The coherence peaks of the diffractogram are further broadened or even smeared out in the presence of a rough boundary or an imperfect alignment. The diffractogram is also sensitive to the uniformity of the structure sizes and fails to show coherence peaks if there is significant size heterogeneity $[15,16]$. Although we chose the corpus callosum to 
minimize the abovementioned effects, our diffractogram still failed to reveal coherent peaks and the shape of the envelope deviated further from the sinc-square function.

Our results show that the length scale of the corpus callosum in adult rats was approximately 10 times greater than the average axonal diameter, about $3 \mu \mathrm{m}$ in electron microscopy. In fact, the measured pattern is affected by experimental parameters and the length scale is characterized by the microstructure size, volume ratio of intracellularto-extracellular compartments and heterogeneity of cellular alignment. Although the measured length scale bears no specific microstructure counterpart, the relative change can still reflect the alteration of the underlying microstructures.

Our results also show that as the brain matured, the diffractogram was stretched to the right progressively, indicating a gradual decrease in the diffusion length scale (Fig. 7C and D). These findings cannot be explained by the change in axonal diameter, which actually increased with age as proven by electron microscopy (Fig. 8). We hypothesize that the length scale measured by diffractogram reflects the average range of water molecular displacement in the microstructure rather than the axonal diameter. In the course of myelination, the transmembrane permeability of axons decreases, causing more restriction to the displacement of water molecules. This turns into a gradual decrease in the measured diffusion length scale in the diffractogram. Our explanation is supported by a theoretical study using a threelayer back-propagation artifact neural network that was conducted by Kuchel and Durrant [17]. They showed that the positions of the nodes in the $q$-space plots moved to larger $q$ values when transmembrane permeability decreased.

Another diffusion MR technique, called $q$-space imaging, has been proposed to probe microstructure sizes $[5,18-$ 20]. Similar to an MR diffusion-diffraction experiment, in this technique, a series of attenuated signals in the $q$-space is obtained. The only difference is that 1D Fourier transform of the spectrum in the $q$-space has to be performed to obtain a 1D profile of the average propagator of molecular diffusion. The root-mean-square displacement is then computed from the average propagator to indicate the microstructure size. The measured spectrum in the $q$-space is either zero filled or extrapolated using a multiexponential decay function to avoid rippling artifacts after Fourier transform. Compared with $q$-space imaging, a diffusion diffractogram interrogates the microstructure size directly in the $q$-space and thus is free from bias arising from different extrapolation models.

As described previously, the diffraction pattern is rarely observed in biologic tissue due to the complex geometry of the microstructures. This article presents the first report on a diffractogram in undissected brains. This diffractogram however requires a more complicated model in which intracellular and extracellular compartments of specific geometry and exchange between the compartments are taken into consideration. The analysis of such a modelbased approach requires simulation and is beyond the scope of the present study. Although this article cannot provide a clear explanation for the rat brain data, it presents a reproducible diffraction pattern in the corpus callosum and a steady change in the diffusion length scale in the course of brain maturation. In correlating with electron microscopy, we speculate that this interesting phenomenon might reflect the underlying myelination of the axons during maturation. Further studies on the effects of myelination on the diffusion length scale can be carried out in animal models of demyelination or dysmyelination.

\section{Conclusions}

Diffusion MRI in the corpus callosum of fixed rat brains observed diffraction-like patterns. The diffusion length scale derived from the diffractogram showed a progressive decrease during brain maturation, which might reflect a gradual decrease in transmembrane permeability due to myelination. The values of this measurement await further investigation in animal models of demyelination or dysmyelination.

\section{Acknowledgments}

This study was supported in part by the National Health Research Institutes (Taiwan; Grant No. NHRI-EX929018EP), the Ministry of Education (Taiwan; Grant for Academic Excellence No. A-91-FA01-2-4) and the National Institutes of Health (USA; Grant No. NIH MH64044).

We thank Dr. Der-Yow Chen and Pai-Feng Yang for their assistance in the animal experiments.

\section{References}

[1] Mansfield P, Grannell PK. NMR ‘diffraction' in solids? J Phys 1973; C6:L422.

[2] Barrall GA, Frydman L, Chingas GC. NMR diffraction and spatial statistics of stationary systems. Science 1992;255:714-7.

[3] Callaghan PT, Eccles CD, Xia Y. NMR microscopy of dynamic displacements: $k$-space and $q$-space imaging. J Phys E Sci Instrum 1988;21:820.

[4] Peled S, Cory DG, Raymond SA, Kirschner DA, Jolesz FA. Water diffusion, $T_{2}$, and compartmentation in frog sciatic nerve. Magn Reson Med 1999;42:911-8.

[5] Chin CL, Wehrli FW, Fan Y, Hwang SN, Schwartz ED, Nissanov J, et al. Assessment of axonal fiber tract architecture in excised rat spinal cord by localized NMR $q$-space imaging: simulations and experimental studies. Magn Reson Med 2004;52:733-40.

[6] Sukstanskii AL, Yablonskiy DA, Ackerman JJH. Effects of permeable boundaries on the diffusion-attenuated MR signal: insights from a one-dimensional model. J Magn Reson 2004;170:56-66.

[7] Kuchel PW, Coy A, Stilbs P. NMR "diffusion-diffraction" of water revealing alignment of erythrocytes in a magnetic field and their dimensions and membrane transport characteristics. Magn Reson Med 1997;37:637-43.

[8] Kuchel PW, Eykyn TR, Regan DG. Measurement of compartment size in $q$-space experiments: Fourier transform of the second derivative. Magn Reson Med 2004;52:907-12.

[9] Torres AM, Michniewicz RJ, Chapman BE, Young GAR, Kuchel PW. Characterisation of erythrocyte shapes and sizes by NMR diffusion- 
diffration of water: correlations with electron micrographs. Magn Reson Imaging 1998;16:423-34.

[10] Torres AM, Taurins AT, Regan DG, Chapman BE, Kuchel PW. Assignment of coherence features in NMR $q$-space plots to particular diffusion modes in erythrocyte suspensions. J Magn Reson 1999;138: $135-43$.

[11] Jiang PC, Yu TY, Perng WC, Hwang LP. Pore-to-pore hopping model for the interpretation of the pulsed gradient spin echo attenuation of water diffusion in cell suspension systems. Biophys J 2001;80:2493-504.

[12] Callaghan PT, Coy A, MacGowan D, Packer KJ, Zelaya FO. Diffraction-like effects in NMR diffusion studies of fluids in porous solids. Nature 1991;351:467-9.

[13] Schmitt P, Griswold MA, Jakob PM, Kotas M, Gulani V, Flentje M, et al. Inversion recovery TrueFISP: quantification of $T_{1}, T_{2}$, and spin density. Magn Reson Med 2004;51:661-7.

[14] Callaghan PT. Principles of nuclear magnetic resonance microscopy. Oxford: Clarendon Press; 1991. p. 328-419.
[15] Chin CL, Wehrli FW, Hwang SN, Jaggard DL, Hackney DB, Wehrli SW. Feasibility of probing boundary morphology of structured materials by 2D NMR $q$-space imaging. J Magn Reson 2003;160: $20-5$.

[16] Topgaard D, Soderman O. Experimental determination of pore shape and size using $q$-space NMR microscopy in the long diffusion-time limit. Magn Reson Imaging 2003;21:69-76.

[17] Kuchel PW, Durrant CJ. Permeability coefficients from NMR $q$-space data: models with unevenly spaced semi-permeable parallel membranes. J Magn Reson 1999;139:258-72.

[18] King MD, Houseman J, Gadian DG, Connelly A. Localized $q$-space imaging of the mouse brain. Magn Reson Med 1997;38:930-7.

[19] Assaf Y, Mayk A, Cohen Y. Displacement imaging of spinal cord using $q$-space diffusion-weighted MRI. Magn Reson Med 2000;44: $713-22$.

[20] Biton IE, Mayk A, Assaf Y, Cohen Y. Structural changes in glutamate cell swelling followed by multiparametric $q$-space diffusion MR of excised rat spinal cord. Magn Reson Imaging 2004;22:661-72. 